
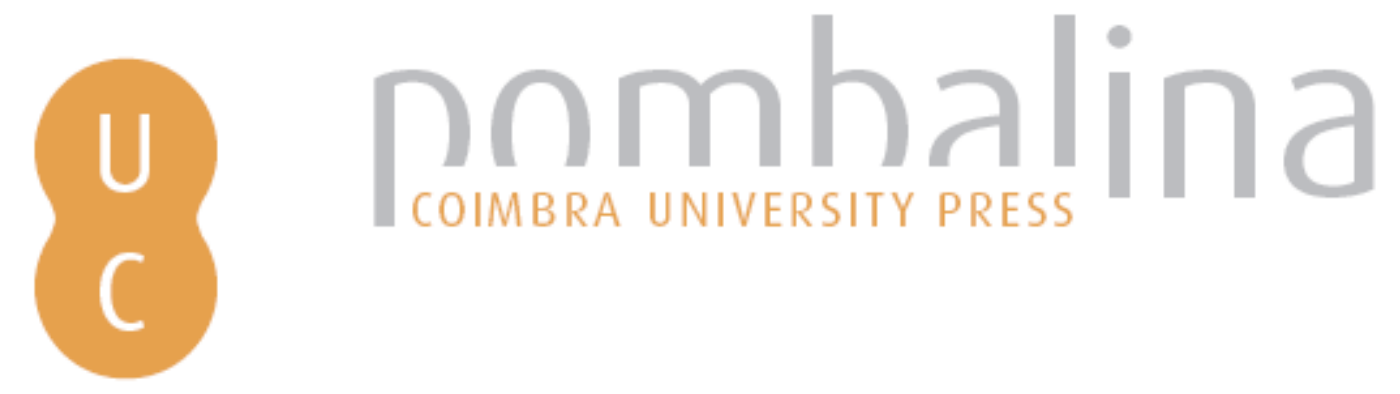

\title{
Modelling the rate of spread of fire: an SDE approach
}

Autor(es): $\quad$ Jovanoski, Zlatko; Sharples, Jason J.; Gill, A. Malcolm; Watt, Simon;

Publicado por: Imprensa da Universidade de Coimbra

URL

persistente: URI:http://hdl.handle.net/10316.2/44579

DOI: $\quad$ DOI:https://doi.org/10.14195/978-989-26-16-506_62

Accessed : $\quad$ 26-Apr-2023 02:52:19

A navegação consulta e descarregamento dos títulos inseridos nas Bibliotecas Digitais UC Digitalis, UC Pombalina e UC Impactum, pressupõem a aceitação plena e sem reservas dos Termos e Condições de Uso destas Bibliotecas Digitais, disponíveis em https://digitalis.uc.pt/pt-pt/termos.

Conforme exposto nos referidos Termos e Condições de Uso, o descarregamento de títulos de acesso restrito requer uma licença válida de autorização devendo o utilizador aceder ao(s) documento(s) a partir de um endereço de IP da instituição detentora da supramencionada licença.

Ao utilizador é apenas permitido o descarregamento para uso pessoal, pelo que o emprego do(s) título(s) descarregado(s) para outro fim, designadamente comercial, carece de autorização do respetivo autor ou editor da obra.

Na medida em que todas as obras da UC Digitalis se encontram protegidas pelo Código do Direito de Autor e Direitos Conexos e demais legislação aplicável, toda a cópia, parcial ou total, deste documento, nos casos em que é legalmente admitida, deverá conter ou fazer-se acompanhar por este aviso.

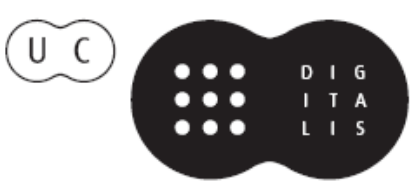




\section{ADVANCES IN}

\section{FOREST FIRE RESEARCH}

\section{8}

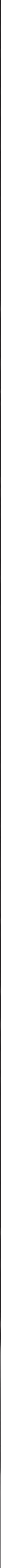




\title{
Modelling the rate of spread of fire: An SDE approach
}

\author{
Zlatko Jovanoski $^{1 *}$; Jason J. Sharples ${ }^{1}$; A. Malcolm Gill ${ }^{2}$; Simon Watt ${ }^{1}$; Harvinder S. Sidhu ${ }^{1}$; Isaac \\ N. Towers ${ }^{1}$; Smaila Sanni ${ }^{1}$ \\ ${ }^{1}$ School of Physical, Environmental and Mathematical Sciences; UNSW Canberra, ACT 2600, \\ Australia.\{z.jovanoski@adfa.edu.au*\} \\ ${ }^{2}$ Fenner School of the Environment and Society; ANU, ACT 0200, Australia
}

\begin{abstract}
From a point source, landscape fires accelerate until they reach a quasi-equilibrium rate of spread. The rate at which a fire accelerates from its ignition affects the time first responders have to attack a fire in its initial stages when it is more easily suppressed. As such, knowledge of the rate of acceleration of a fire from ignition can be valuable from a fire management perspective. However, the majority of studies in wildland fire science have been dedicated to development of models for the quasi-equilibrium rate of spread attained by the fire after its acceleration phase. Comparatively little attention has been given to the development of models that specifically account for the growth phase of a fires development.

The rate of acceleration depends on many factors including variations in ambient and induced wind speed and direction, variation in moisture content of the fuel, fuel stratification and slope variation. Present models of fire growth from a point ignition are expressed as deterministic algebraic equations, thereby neglecting variability. The numerous variables involved make predictions of rate of spread from a point source very difficult.

In this paper we consider two approaches to model the acceleration phase of a fire. The first considers fitting a sigmoidal (logistic) function to experimental data using a nonlinear regression procedure. In the second approach we propose the use of stochastic differential equations to investigate the growth of a fire to quasi-equilibrium. In addition to providing a more realistic portrayal of the time series data relating to fire growth, this second approach allows for better discrimination of the mechanisms driving the growth phase of fire spread.

The models are assessed by appealing to observations of experimental fire growth. Specifically the data relate to fires growing from a point ignition under the influence of a uniform wind. The results indicate that both approaches can provide an accurate representation of the observed data, but that the approach based on stochastic differential equations yields $95 \%$ prediction bounds that are narrower than those obtained from the nonlinear regression. The difference in prediction bounds indicates that the way stochasticity is incorporated into fire growth models has implications for how models inform decisions about the likelihood of a fire selfextinguishing before it reaches quasi-equilibrium, and the magnitude of the rates of spread it is likely to exhibit during the initial stages of growth.
\end{abstract}

Keywords: Fire growth; fire acceleration; rate of spread; nonlinear curve-fitting; stochastic differential equation

\section{Introduction}

Bushfires are a significant environmental problem. In the last decade this has become increasingly apparent, with several major conflagrations causing extensive loss of life and property, and considerable environmental damage. Regardless of the ultimate size and intensity of a bushfire, all fires start small - often originating as a point ignition, or something akin to a point ignition, and growing in a manner that is governed by the ambient environmental conditions. In addition, under certain conditions a bushfire will deposit firebrands ahead of the main front, igniting spot-fires, which can also be viewed as evolving from a point source.

Landscape fires originating from a point source accelerate until they reach a quasi-equilibrium rate of spread. This behaviour has also been confirmed in laboratory experiments (McAlpine \& Wakimoto 
1991). Attacking fires while they are in their acceleration phase, before they have attained their potential quasi-steady rate of spread, provides a greater chance of suppression success (Cheney \& Gould 1997). As such, there are good reasons to understand the likely behaviour of a fire during its acceleration phase, and mathematical modelling provides a means to improve this understanding. However, in comparison with the vast literature that deals with modelling the quasi-equilibrium rate of spread, there is little that specifically considers this acceleration phase of a fires development (Cruz et al. 2015). The way a fire develops during its acceleration phase depends on the local environmental conditions, including variations in ambient and induced wind speed and direction, and variations in moisture content and structure of the fuel. Even when a fire attains quasi-equilibrium state, its rate of spread can still exhibit considerable variation about a mean value.

However, present models of fire growth from ignition are expressed as deterministic algebraic equations, thereby neglecting variability. Parameters in such models are estimated using nonlinear regression of empirical data, and once determined they are considered as fixed; separate estimates are required for different fuel types and environmental conditions. Any resulting model lacks flexibility and should not be used in circumstances where the fuel and environmental conditions are heterogeneous.

Moreover, interactions between winds, terrain, different vegetation types and structures means that the conditions affecting the initial growth of a fire (and its longer-term propagation) can never be adequately quantified in solely deterministic terms. Coupling between the fire and the atmosphere further confound this situation. The intrinsic variability of the factors affecting the initial growth of a fire means that deterministic modelling approaches are ill-suited to modelling this aspect of fire spread. Indeed, such approaches gloss over the detailed dynamics, which are inherently stochastic in nature due to the turbulent conditions under which fires develop. Recent advances in stochastic dynamical systems theory and their applications, as well as in our understanding of dynamic fire behaviour mean that we are now able to explicitly address the acceleration phase (from a point ignition) of fire growth in terms of stochastic dynamic systems.

The models we propose in this paper appear to be the first to consider the acceleration phase of a fire's growth as a stochastic dynamical system. Such an approach permits a probabilistic interpretation of fire growth, which allows for estimation of worst-case and mean-case scenarios. Also, by explicitly accounting for the intrinsic variability of fire spread and the environmental conditions that drive it, our stochastic approach has the potential to provide greater insights into the physical processes governing fire growth, particularly in its acceleration phase.

\section{Rate of spread data}

The rate of spread (ROS) data used in this study are taken from McAlpine \& Wakimoto (1991). The data were extracted using G3DATA (Frantz 2000) and are reproduced in Figure 1. The data set represents five replicates of the ROS observed in a wind tunnel experiment. In each case the fuel load was $26.3 \mathrm{~kg} / \mathrm{m}^{3}$ and burned under the influence of a wind speed of $4.8 \mathrm{~km} /$ hour. The temperature $\left(26.7^{\circ} \mathrm{C}\right)$ and humidity $(80 \%)$ were controlled for. The fuel moisture content was measured just prior to burning, and the mean of the five replicates was $8.62 \%$ (standard deviation of 0.57 ). It is of fundamental interest that even with carefully controlled conditions and a fixed wind speed, the variation in ROS can be substantial. As already mentioned, this variation may be due to differing fuel moisture content, variability in the packing of the fuel bed for each experimental run (Finney 2004), and turbulence induced by the fire itself. We treat each of the replicates in Figure 1 as independent data sets. 


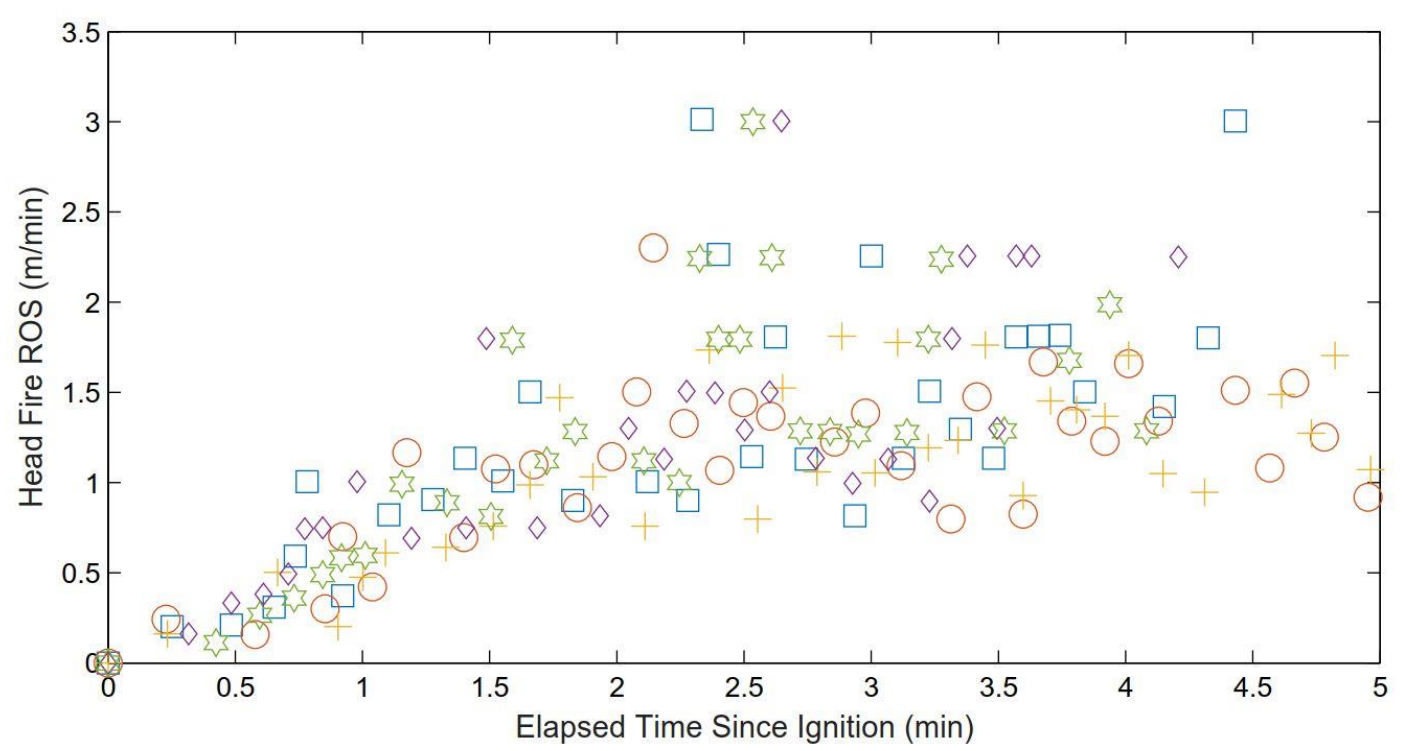

Figure 1 - Observed rate of spread for a pine needle fuel bed, with a bulk density of $26.3 \mathrm{~kg} / \mathrm{m}^{3}$ and burned at a wind speed of $4.8 \mathrm{~km} / \mathrm{hr}$. Five replications are shown, each symbol (square, circle, etc.) being a separate replication.

(Figure recreated from McAlpine \& Wakimoto (1991).)

\section{Mathematical modelling of the rate of spread}

\subsection{Nonlinear regression}

Typically, a model for the ROS, $R(t)$, is obtained by postulating a functional form with a set of unknown parameters that need to be determined. The shape of the functional form is driven by the data. McAlpine \& Wakimoto (1991) provide a summary of some well-known models that are relevant for different environmental conditions and fuel types. These include exponential-type models $R(t)=R_{e} \exp (-a / t)$ and $R(t)=R_{e}(1-\exp (-a t))$, and power-law models $R(t)=\beta t^{\alpha}$. In all cases $\alpha, \beta$ and $R_{e}$ are constants that need to be determined. In the exponential-type models, $R(t) \rightarrow R_{e}$ as $t \rightarrow \infty$, where $R_{e}$ is the asymptotic or quasi-equilibrium ROS. The power-law model never attains a quasi-equilibrium state.

Considering the data in Figure 1, it appears that there is an initial rapid increase in the ROS until reaching an inflection point, after which the acceleration begins to decrease, asymptotically approaching the quasi-equilibrium ROS. To describe this behaviour, we propose a sigmoid (logistic) function:

$$
R(t)=\frac{R_{0} R_{e}}{\left(R_{0}+\left(R_{e}-R_{0}\right) \exp (-a t)\right)}+\epsilon_{i}, \quad i=1, \ldots, n
$$

for $n$ data points $\left(t_{i}, R\left(t_{i}\right)\right)$. Here $R_{0}$ is the initial value of the ROS, the quasi-equilibrium value is $R_{e}$ and $a$ is the rate of increase of the ROS. The $\epsilon_{i}$ are independent and normally distributed errors. The basic assumptions of regression analysis are assumed except constant variance. The latter is justified since the variability in the data tends to increase with increasing ROS.

The aim is to estimate the three parameters, $R_{0}, R_{e}$ and $a$, by implementing a weighted nonlinear least-squares method to minimise the sum of the weighted square of the errors (or weighted residuals) between the observed $R\left(t_{i}\right)$ and the curve-fitted values for each $t_{i}$. The weighted sum of squares is 


$$
L_{w}\left(R_{0}, R_{e}, a\right)=\sum_{i=1}^{n} w_{i}\left(R\left(t_{i}\right)-\frac{R_{0} R_{e}}{\left(R_{0}+\left(R_{e}-R_{0}\right) \exp (-a t)\right)}\right)^{2}
$$

where $w_{i}$ are the weights. The weights determine how much each $R\left(t_{i}\right)$ value influences the final parameter estimates. Here the residuals are weighted using a bi-square weighting scheme. This method minimises a weighted sum of squares, where the weight given to each data point depends on how far the point is from the fitted line. Points near the line get full weight. Points farther from the line get reduced weight. Points that are farther from the line than would be expected by random chance get zero weight. The bi-square weights are given by

$$
w_{i}= \begin{cases}\left(1-\left(u_{i}\right)^{2}\right)^{2}, & \left|u_{i}\right|<1 \\ 0, & \left|u_{i}\right| \geq 1,\end{cases}
$$

where the $u_{i}$ are the standardised adjusted residuals. Robust fitting with bi-square weights uses an iteratively re-weighted least-squares algorithm.

The minimisation of $L_{w}$ with respect to the parameters must be carried out iteratively. The Levenberg-Marquardt method is a standard technique for solving nonlinear-least squares problems (Rowlings et al. 1998). The Levenberg-Marquardt curve-fitting method is a combination of two minimisation methods: the gradient descent method and the Gauss Newton method. In the gradient descent method, the sum of the squared errors is reduced by updating the parameters in the steepestdescent direction. In the Gauss-Newton method, the sum of the squared errors is reduced by assuming that the least squares function is locally quadratic and finding the minimum of the quadratic. The Levenberg-Marquardt method acts more like a gradient-descent method when the parameters are far from their optimal value and acts more like the Gauss-Newton method when the parameters are close to their optimal value. Both options are available in MATLAB.

\subsection{A stochastic differential equation approach}

An alternative to nonlinear regression is to reformulate the model as a differential equation (DE), specifically as a stochastic differential equation (SDE). For a dynamic process such as the spread of a fire it seems more natural to employ a DE or an SDE. Using an SDE allows for the inclusion of local environmental variability that might be due to heterogeneous fuels or changes in wind direction and strength.

With reference to Figure 1, during the early development of the fire, $R^{\prime}(t) \propto R(t)$, then as $R(t) \rightarrow R_{e}, R^{\prime}(t) \rightarrow 0$. This behaviour is captured by the logistic DE. The stochastic version of the logistic DE written in the Ito form is (Gardiner 2009),

$$
\mathrm{dR}=a R\left(1-\frac{R}{R_{e}}\right) d t+\sigma R d W, \quad R(0)=R_{0}
$$

where the parameters $R_{0}, R_{e}$ and $a$ have the same meaning as before. The first term on the righthand side is the drift and the second term is the stochastic term, often called the diffusion. The parameter $\sigma$ is the strength of the local environmental variability and $W(t)$ is a standard Wiener process with expected value $\mathbb{E}[W(t)]=0$ and variance $\mathbb{V}[W(t)]=t$. The appearance of $R(t)$ in the stochastic term assumes that the variance increases with increase in the ROS. For $\sigma=0$ the solution to the resulting (deterministic) ODE is the sigmoidal curve (1). This provides a basis for comparing the two modelling approaches. 
It is worth noting that equation (2) is related to the power-law nonlinear model of Dold \& Zinoviev (2009), for the modelling of fire eruptions. Under certain conditions the rate of change of the ROS satisfies

$$
\frac{\mathrm{dR}}{\mathrm{dt}}=a R\left(1-\left(\frac{R}{R_{e}}\right)^{\frac{1}{v}-1}\right)
$$

with $v<1$. Setting $v=2$, we arrive at equation (2). For this model, as with the equation (2), there exist two equilibrium points (in the absence of noise), namely $R(t)=0$ (unstable) and $R(t)=R_{e}$ (stable). This means any slight perturbation off the $R(t)=0$ equilibrium state, the ROS will accelerate towards the $R(t)=R_{e}$ state.

It should be further noted that according to equation (2), the acceleration starts off small, increasing until reaching the maximum acceleration of $R_{e} / 2$ after which the acceleration begins to decrease approaching zero as $R(t) \rightarrow R_{e}$. This is reflected in the solution curve of $R(t)$ as a concave up function during the initial phase of fire spread, followed by a change in concavity at the inflection point at $R_{e} / 2$. This is consistent with the choice of the nonlinear regression curve of equation (1).

\section{Comparing the methods}

For illustrative purposes we fit the curve to a single replicate of the ROS data (the squares) in Figure 1. The null hypothesis is tha $R_{0}, R_{e}$ and $a$ are zero, compared to the alternate hypothesis that $R_{0}, R_{e}$ and $a$ is non-zero. The estimated parameters including a $95 \%$ confidence interval (CI) is shown in Table 1.

For the ROS data, the estimated values and their CIs indicate there is statistical evidence to reject the null hypothesis, except for $R_{0}$. This is problematic as it implies the model is inadequate. To check this the integral of equation (1) was fitted to the measured distance travelled by the fire front from the time of ignition. On this ocassion all of the estimated parameters are significant (see Table 1).

Table 1 - Nonlinear curve-fitting to a single replicate (square symbol) to both the ROS and distance travelled by head of fire front

\begin{tabular}{ccccccc}
\hline \hline & \multicolumn{3}{c}{ Estimated Parameters } & & & \\
\cline { 2 - 4 } Data type & $R_{0}$ & $R_{e}$ & $a$ & RMSE & $\mathrm{R}^{2}$ & Adj R $^{2}$ \\
\hline ROS & 0.203 & 1.576 & 1.622 & 0.445 & 0.648 & 0.623 \\
& $(-0.109,0.516)$ & $(1.248,1.904)$ & $(0.135,3.109)$ & & & \\
\hline Distance & 0.170 & 1.416 & 1.927 & 0.051 & 0.999 & 0.999 \\
& $(0.121,0.219)$ & $(1.366,1.466)$ & $(1.567,2.288)$ & & & \\
\hline ROS & 0.170 & 1.550 & 1.827 & 0.4263 & 0.665 & 0.654 \\
(refitted) & & $(1.305,1.809)$ & $(1.165,2.485)$ & & & \\
\hline
\end{tabular}

The reason for the difference is that the ROS, which is derived (backward differecing) from the observed distance data, is highly variable. There is an order of magnitude difference in the Root Mean Square Error (RMSE) - the sandard deviation of the weighted residuals - a measure of spread of the difference between the predicted and observed values. The estimated $\mathrm{R}^{2}$ indicates that about $65 \%$ of the variability in the ROS data is accounted for by the fitted curve and a near perfect fit for the distance data. Furthermore, plots of weighted residuals (not shown) indicate homoscedasticity of the variance and a lack of correlation in the errors.

Our aim is to compare the nonlinear regression with that of the SDE. We therefore fix the $R_{0}$ value obtained from the distance data and determined the other two parameters for the ROS data. The 
parameter estimates for the newly fitted nonlinear regression is also shown in Table 1. The CI for $R_{e}$ and $a$ are narrower, a slight reduction in RMSE and a slight increase in $\mathrm{R}^{2}$ which all amounts to an improvemnt with the refitted nonlinear curve (one less parameter to fit). A plot of the nonlinear regression curve is portrayed in Figure 2. Also shown is the 95\% prediction interval (PI) that represents an interval for a new observation of the ROS, conditioned on the observed data.

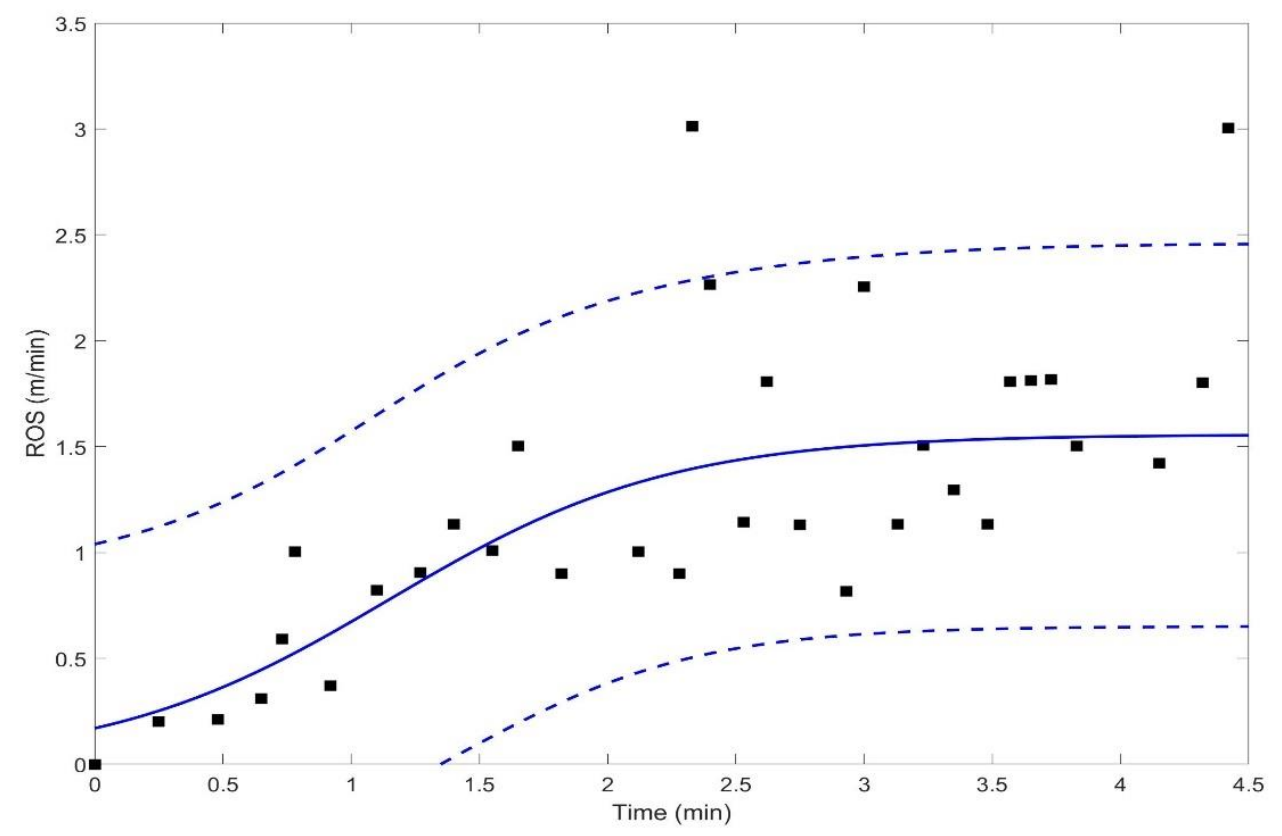

Figure 2 - Plot of the nonlinear regression curve to the ROS data including a 95\% prediction interval for a new observation of the ROS.

The solution to (2) typically requires a numerical approach, the simplest of these is the EulerMaruyama algorithm (Higham 2001). At the heart of this algorithm is the following discretisation of the SDE

$$
R_{i+1}=R_{i}+a R_{i}\left(1-\frac{R_{i}}{R_{e}}\right) \Delta t_{i}+\sigma R_{i} \Delta W_{i}
$$

where $\Delta t_{i}=t_{i+1}-t_{i}, \Delta W_{i}=W_{i+1}-W_{i}=\sqrt{\Delta t_{i}} \xi_{i}$ and $\xi_{i} \sim N(0,1)$. The numerical solution to the SDE is a single realisation, but what is of importance is to determine the expected value and variance of a large number of such realisations.

To solve equation (2) we must specify four parameters: $R_{0}, R_{e}, a$ and $\sigma$. Strictly speaking we should estimate these parameters by using maximum-likelihood methods or other related techniques directly to (2) (Kloeden et al. 1994). Here, however, we make use of the values already determined previously, and our tasks remains to find $\sigma$. This is done by searching through a range of values of $\sigma$ for which the estimated variance $\mathbb{V}[R(t)]$ (estimated at $t \approx 4.5 \mathrm{~min}$ ) is equal to the MSE (square of the RMSE). This is justifiable if we seek to make a comparison between (1) and (2).

To estimate both $\mathbb{E}[R(t)]$ and variance $\mathbb{V}[R(t)]$ we employ an Monte Carlo approach with a large number of realisations. Figure 3 depicts the results of the 100,000 realisations. The figure shows the expected ROS with a 95\% PI. Requiring $\mathbb{V}[R(t)] \approx M S E$ yields $\sigma=0.545$. It is clear that almost all of the data resides within the $95 \%$ PI, suggesting that the proposed model provides a good description for the observed ROS. 


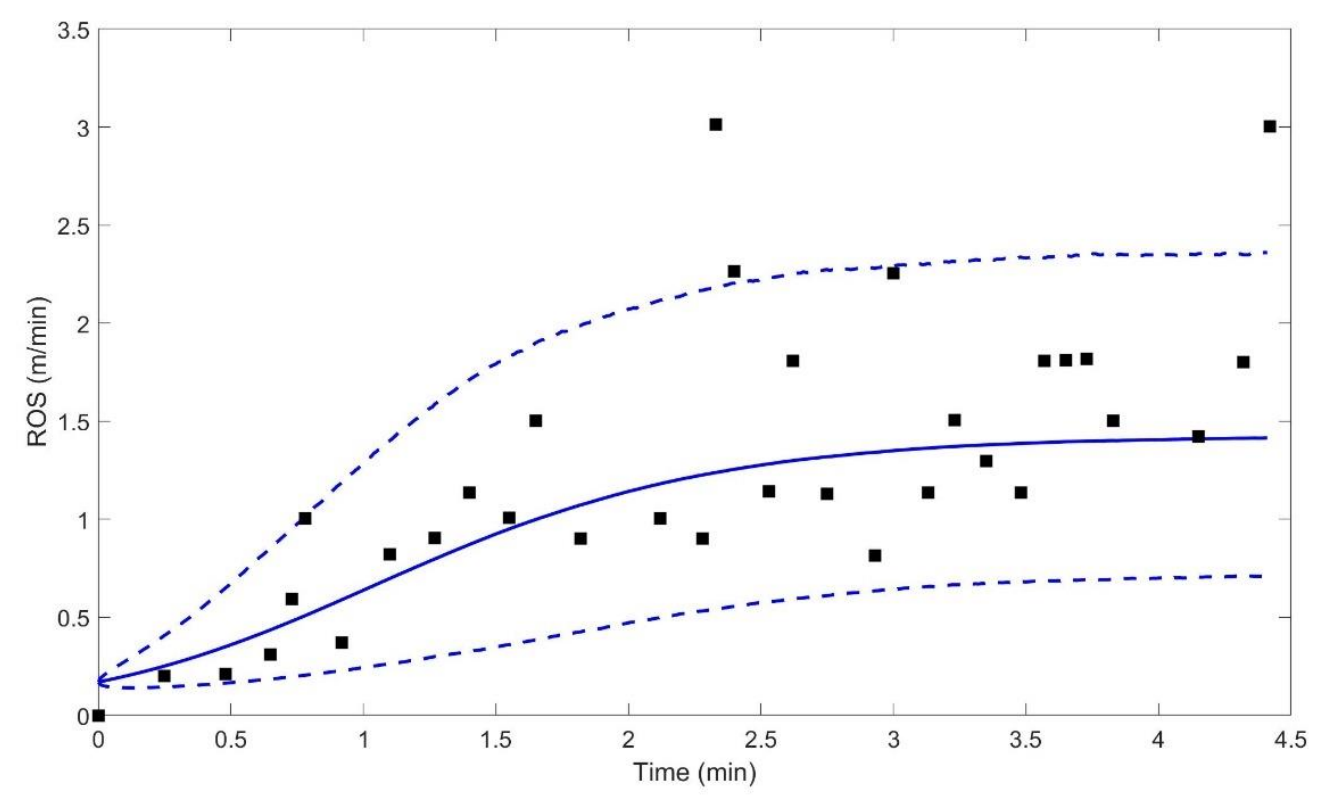

Figure 3 - Plot of the expected ROS based on 100,000 realisations of the SDE. Also shown is the 95\% prediction interval for a new realisation of the $S D E$. Here $t=0.01$.

Over the time domain relevant for the duration of the experiment, the lower of the $95 \%$ PI offers practical information about the likelihood of the fire self-extinguishing, often referred to as a 'go/nogo' threshold. The closer the lower prediction interval is to zero, the more likely the fire will selfextinguish. The upper prediction interval provides important information for fire management, as it relates to the potential for rapid initial growth of a fire in the landscape. Knowledge of the upper prediction interval can therefore give fire managers a better idea of the operational window for initial response once an ignition occurs, thus enabling more informed deployment of personnel and assets to head off a fire during its initial stages.

It is of interest to note that both of the PIs for the SDE model are roughly the same as for the nonlinear regression - the variance for both models was set to be equal once quasi-equilibrium was reached. Since they both have the same variance, any differences must be intrinsic to the way stochasticity is accounted for in each model. The major difference appears in the intial acceleration of the fire. Based on the width of the PI, the nonlinear regression predicts that self-extingishing is more likely during the first 1 min since ignition, compared to that of the SDE. The SDE on the other hand shows a rather narrower PI which increases over time. The chances of the fire to self-extinguish for the same set of parameters is greatly reduced. The result imply that a fire whose behaviour is more in accord with the SDE model is less likely to self-extinguish, and has the potential to exhibit higher rates of spread.

Computing a large number of realisations allows us to generate statistics concerning fire spread. Figure 4 shows a frequency distribution of the ROS at four different times: $1 \mathrm{~min}, 2 \mathrm{~min}, 3 \mathrm{~min}$ and 4 min. Superimposed is a continuous curve obtained via kernel smoothing to help aid in identifying the location of the distribution and the skewness that is apparent at earlier times. The distribution is narrow at earlier times, the distribution continues to evolve and by $t \approx 3 \mathrm{~min}$ the frequency distribution remains relatively unchanged, having achieved a quasi-equilibrium state. From this we can estimate the probability that the ROS is larger than a particular value, or lies within some range of values, at any time $t$ after ignition. This is in contrast to the nonlinear regression foe which the distrubution is stationary for all times. 

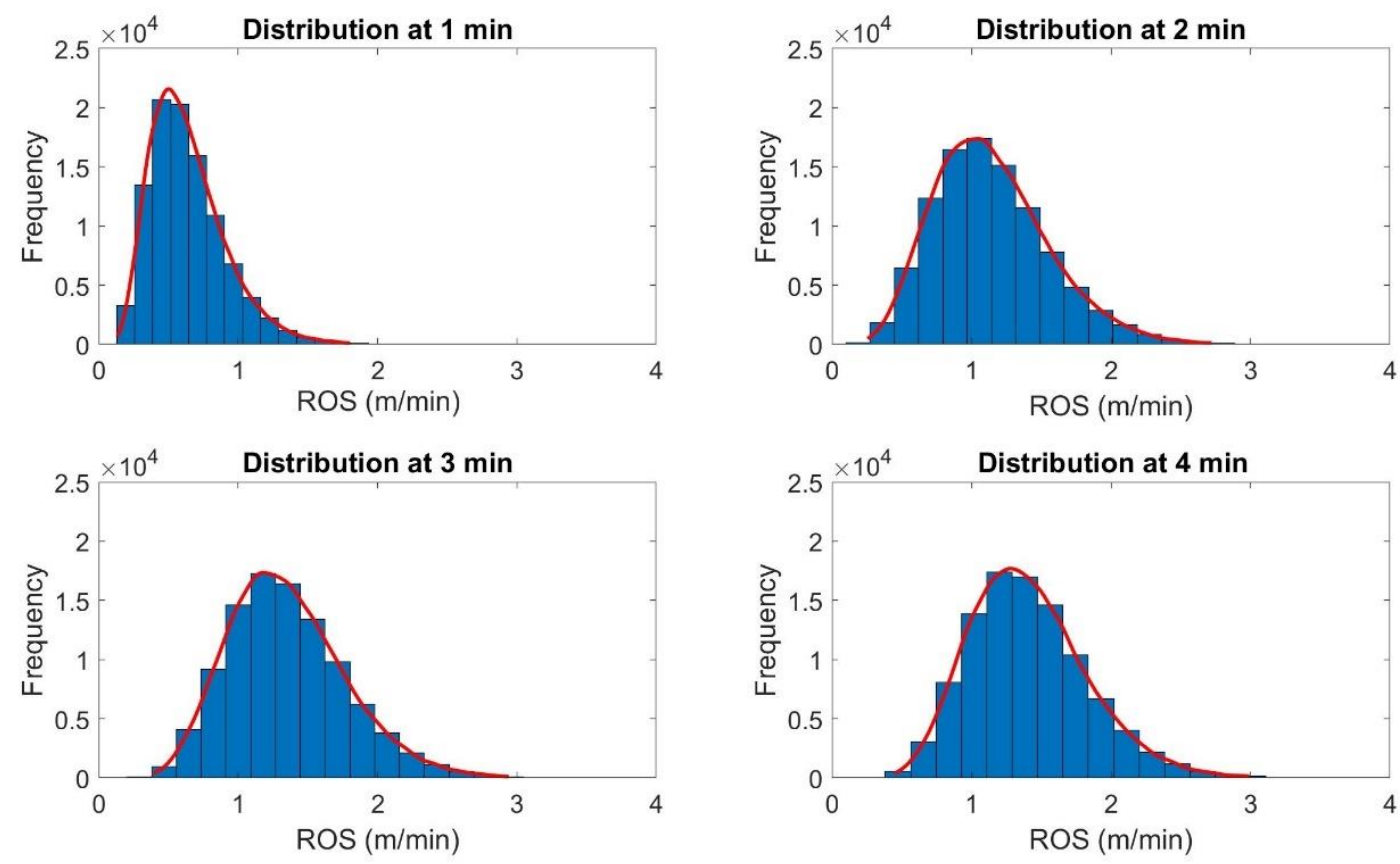

Figure 4 - The evolution of the frequency distribution for the ROS based on 100,000 realisations.

\section{Heterogeneity - preliminary analysis}

Equation (2) is a first step toward modelling the fire growth from ignition using an SDE. The manner by which stochasticity is added is a modelling issue that deserves further inquiry. Equation (2) incorporates stochasticity by aggregating the effect of growth and self-limiting of the spread, both of which are affected by local environmental variability, into a single term.

To use the nonlinear regression (1) in making predictions requires that the parameters be determined and kept fixed for a given set of environmental conditions. This means for a change in wind speed requires equation (1) be refitted, likwise if excelsior fuel is used instead of pine needles. The SDE approach (2) allows slightly more flexibility, but here too the model is inadequate as it cannot discriminate how different conditions can impact fire growth and accelaration - such as different fuel types, changes in wind strength and direction and variability in moisture content, etc. This limits the usefulness and application of both approaches.

However, some progress cna be made by treating some of the parameters as state variables. Suppose that the local quasi-equilrium rate of spread $R_{e}(t)$ is now a state variable and is a function of the wind velocity. We consider the effect of changes in wind speed and direction. The simplest of such models is described by a stochastic process

$$
\begin{gathered}
\frac{\mathrm{dR}}{\mathrm{dt}}=a R\left(1-\frac{R}{R_{e}}\right) \\
d R_{e}(t)=\sigma d W
\end{gathered}
$$

Here $d W(t)$ represents a change in the local wind direction and strength; $d W(t)>0$ helps to increase $R_{e}$, while $d W(t)<0$ to decrease $R_{e}$. The exact solution is $R_{e}(t)=R_{e}(0)+\sigma W(t)$ with $\mathbb{E}\left[R_{e}(t)\right]=R_{e}(0)$ and $\mathbb{V}\left[R_{e}(t)\right]=\sigma t$. 


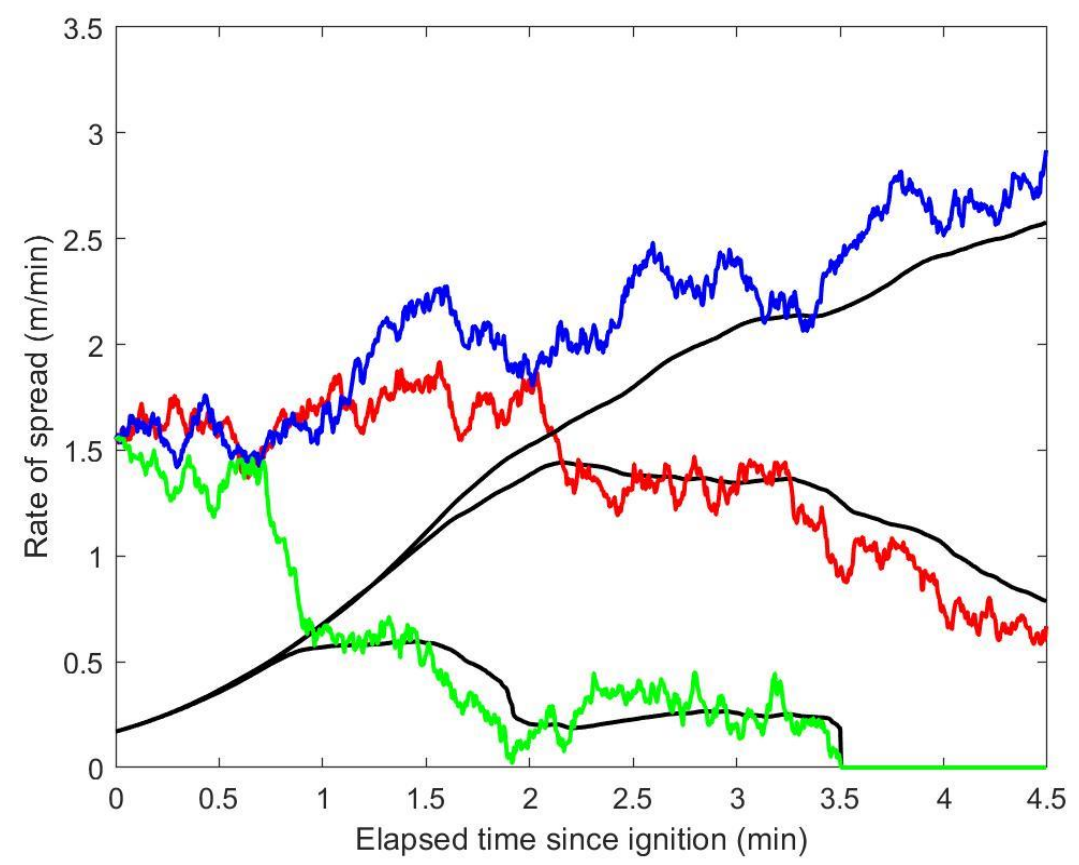

Figure 5 - Plot of three different realisations of the local quasi-equilibrium and the solution (black) for the ROS based on model (3). One realsistion is seen to self-extinguish (green).

Figure 5 depicts three realisations of equation (3) with $R_{e}(0)=1.550$. One realisation (blue) is the effect of a number of favourable wind changes that have increased the local $R_{e}(t)$ thus increasing $R(t)$. Due to a series of unfavourable wind changes in another realisation (green) the $R_{e}(t)$ is seen to have reached zero, and according to equation (3), the fire has self-extinguished.

We are now in a position to estimate the probablity that a fire may self-extinguish. Table 2 summarises the results based on 10,000 simulations of equation (3) for different levels of noise intensity. The probability increases as the ratio $R_{e}(0) / \sigma$ decreases.

Table 2 - Estimate of the probability to self-extinguish based on 10,000 realisations of equation (3)

\begin{tabular}{|c||c|c|c|c|}
\hline $\boldsymbol{\sigma}$ & 0.2 & 0.4 & 0.6 & 0.8 \\
\hline Probability & $2.6 \times 10^{-4}$ & $6.8 \times 10^{-2}$ & 0.22 & 0.36 \\
\hline
\end{tabular}

The idea that the ROS depends on the energetic ratio balance between the heat released by combustion and the energy necessary for fuel ignition is well known (Viegas, 2004). Certainly the energy required for ignition of moist fuel is greater than that of dry fuel, thus mosit fuel will have a dampening effect on $R_{e}$. Based on experiments, Rossa (2017) developed an empirical model for $R_{e}$ that is a power-law of the moisture content of the fuel. This could be incorporated into equation (3) to model the effect of a fuel with variable moisture content on the ROS as the fire develops.

Lastly, another important effect on the ROS is the curvature of the fire front. For a point-source ignition, the fire front curvature is large and as the fuel burns the radius of the fire front increases, the curvature decreases, until at such time the expanding ring of fire essentially behaves as a line fire. Point-source ignition takes a longer time than a line ignition to accelerate to $R_{e}$ (McRae 1999). However, differences can be variable and depend on the types of fuels (Finney \& McAllister 2011). Weber (1989) developed a theoretical framework for the ROS from a point-source ignition, and the conjoining of this model with that of equation (3) may be used to model the evolution of a point-source ignition to a line fire subject to variable environmental conditions. 


\section{Conclusion}

In developing models for the spread of fire it is customary to fit the data based on some prescribed functional form. Such methods provide good descriptions of the time evolution of the mean ROS from time of ignition. The aim is to obtain parameter estimates and relevant CIs of the fixed parameters for the spread rate. Alternatively, an SDE formulation is a natural way to describe the rate of change of the ROS, a quantity that can be experimentally measured. Carrying out a large number of simulations enables the construction of an empirical probability density function for the ROS, from which statistical quantities can be determined. This has practical implications for the management of fires.

The model we propose explicitly incorporates the stochastic dynamics of fire spread and environmental variability. Through our model we seek to provide a more appropriate method for assessing the likely development of fires in the landscape. It is expected that the model can be used to better inform the decisions that need to be made by fire managers through provision of a probabilistic framework that acknowledges the uncertainties inherent in fire spread.

The SDE formulation for the spread rate is particularly attractive as it provides a way to explicitly involve a fire's history (Viegas 2004). This could be accomplished by using either a distributed timedelay in the spread rate, which involves a weighted average of the spread rate taken over all previous times, or as a discrete time-lag due to the process of pyrogenesis (Dold \& Zionviev 2009). This line of research is currently being investigated.

\section{References}

Cheney, N. P. \& Gould, J. S. (1997), Fire growth and acceleration, International Journal of Wildland Fire $7,1-5$.

Cruz, M. G., Gould, J. S., Alexander, M. E., Sullivan, A. L., McCaw, W. L. \& Matthews, S. (2015), A guide to rate of fire spread models for Australian vegetation, Technical report, CSIRO Land and Water Flagship, Canberra, ACT, and AFAC, Melbourne, Vic, 123pp.

Dold, J. W. \& Zionviev, A. (2009), Fire eruption through intensity and spread-rate interaction mediated by flow attachment, Combustion Theory and Modelling 13, 763-793.

Finney, M. A. (2004), Farsite: Fire area simulator - model development and evaluation, Technical report, USDA For. Serv., Rocky Mt. Res. Stn., Ogden, UT. Res. Pap. RMRS-RP-4 Revised. 47 p.

Finney, M. A. \& McAllister, S. S. (2011), A review of fire interactions and mass fires, Journal of Combustion 2011, article ID 548328 (14 pages).

Frantz, J. (2000), 'G3DATA', http://www.frantz.fi/software/g3data.php. [Online: accessed March 20, 2017].

Gardiner, C. (2009), Stochastic Methods: A handbook for the natural and social sciences, SpringerVerlag, Berlin.

Higham, D. J. (2001), An algorithmic introduction to numerical simulation of stochastic differential equations, SIAM. Review 43, 525-546.

Kloeden, P. E., Platen, E. \& Schurz, H. (1994), Numerical solution of SDE through computer experiments, Springer-Verlag, Berlin.

McAlpine, R. S. \& Wakimoto, R. H. (1991), The acceleration of fire from point source to equilibrium spread, Forest Science 37, 1314-1337.

McRae, D. J. (1999), Point-source fire growth in jack pine slash, International Journal of Wildland Fire 9, $65-77$.

Rossa, C. G. (2017), The effect of fuel moisture content on the spread rate of forest fires in the absence of wind or slope, International Journal of Wildland Fire 26, $24-31$. 
Rowlings, J. O., Pantula, S. G. \& Dickey, D. A. (1998), Applied regression analysis: A research tool, 2 edn, Springer-Verlag, New York.

Viegas, D. X. (2004), On the existence of a steady state regime for slope and wind driven fires, International Journal of Wildland Fire 13, 101-117.

Weber, R. O. (1989), Analytic models for fire spread due to radiation, Combustion and Flame 78, 398-408. 